\title{
Interactive Gaps in Teleological Service Encounters: An Abstract
}

\author{
Göran Svensson and Carmen Padin
}

\begin{abstract}
This study examines how the teleological actions of service providers and service receivers in complaint handling relate to negative emotions after critical incidents have occurred on the one hand, and to perceived justice on the other. Teleological actions may be either ad hoc and present-based, predetermined and past-based; or goal-directed and future-based. The objective here is to describe service receivers' teleological actions and their perceptions of service providers' teleological actions in complaint handling after critical incidents have occurred in service encounters. The study focuses on the learning and adaptive learning curves in service encounters, as suggested by Padin et al. (2015). It also provides insights into interactive gaps and indications of cause-and-effect in teleological service encounters. A qualitative method was used to study teleological actions in service encounters. The focus is on after critical incidents have occurred that cause negative emotions in service encounters. The study offers a valuable contribution in relation to calls in the literature for additional perspectives and approaches on the interface between negative emotions and perceived justice in service encounters (e.g., Bagozzi et al. 1999; Scott et al. 2014; Svaeri et al. 2011; Svensson and Padin 2013). A different perspective is applied, using teleological actions in the context of learning and adaptive curves that may (or may not) take into consideration past actions in the present for future actions, after critical incidents have occurred that cause negative emotions in service encounters. It is concluded that taking into account a service receiver's teleological learning curve, in relation to a service provider's teleological adaptive curve provides meaningful guidance and a structure for assessing and managing critical incidents and negative emotions in service encounters.
\end{abstract}

References Available Upon Request

\author{
G. Svensson ( $\square)$ \\ Kristiania University College, Oslo, Norway \\ e-mail: goran.svensson@kristiania.no \\ C. Padin \\ Vigo University, Vigo, Spain \\ e-mail: padin@uvigo.es
}

